\title{
Binomial Hadamard Series and Inequalities over the Spectra of a Strongly Regular Graph
}

\author{
Luís Vieira \\ Department of Civil Engineering, University of Porto, Porto, Portugal \\ Email: lvieira@fe.up.pt.
}

How to cite this paper: Vieira, L. (2018) Binomial Hadamard Series and Inequalities over the Spectra of a Strongly Regular Graph. Applied Mathematics, 9, 1055-1071. https://doi.org/10.4236/am.2018.99071

Received: July 27, 2018

Accepted: September 25, 2018

Published: September 28, 2018

Copyright $\odot 2018$ by author and Scientific Research Publishing Inc. This work is licensed under the Creative Commons Attribution International License (CC BY 4.0).

http://creativecommons.org/licenses/by/4.0/

\begin{abstract}
Let $G$ be a primitive strongly regular graph of order $n$ and $A$ is adjacency matrix. In this paper we first associate to $A$ a real 3-dimensional Euclidean Jordan algebra $\mathcal{A}$ with rank three spanned by $I_{n}$ and the natural powers of $A$ that is a subalgebra of the Euclidean Jordan algebra of symmetric matrix of order $n$. Next we consider a basis $\mathcal{B}$ that is a Jordan frame of $\mathcal{A}$. Finally, by an algebraic asymptotic analysis of the second spectral decomposition of some Hadamard series associated to $A$ we establish some inequalities over the spectra and over the parameters of a strongly regular graph.
\end{abstract}

\section{Keywords}

Euclidean Jordan Algebras, Graph Theory, Strongly Regular Graphs

\section{Introduction}

Good surveys on Euclidean Jordan algebras can be found in books such as $A$ Taste of Jordan Algebras of Kevin McCrimmon [1], Analysis on Symmetric Cones of Faraut and Korányi [2] and in the Koecher's Minnesota Notes on Jordan Algebras and Their Applications [3]. Euclidean Jordan algebras become a good tool for the analysis of primal dual interior point methods [4] [5] [6] and [7]. But they have also a lot of applications on other branches of mathematics namely on the formalism of quantum mechanics [8], on combinatorics [9]-[15], and on statistics [16]. Recently, many authors had extended some properties of matrix theory to the Euclidean Jordan Algebras, see for example [17] [18] and [19].

In this paper we analyse the spectra of Hadamard series associated to the adjacency matrix of a strongly regular graph to deduce asymptotic inequalities on the spectra and on the parameters of a strongly regular graph in the environment of Euclidean Jordan algebras. 
The plan of this paper is as follows. In Section 2 we expose the principal definitions and results on Euclidean Jordan algebras. Next, in Section 3 we present the more relevant definitions and results on strongly regular graphs necessary for a clear exposition of this paper. Finally, in Section 4 we associate a three dimensional real Euclidean Jordan algebra $A$ to a strongly regular graph and next we establish asymptotic inequalities on the spectra and on the parameters of a strongly regular graph, see (10) and (20) of Theorem 4 and 5 respectively.

\section{A Short Introduction to Euclidean Jordan Algebras}

Herein, we make an introduction to Euclidean Jordan algebras and present the definitions and the more relevant results needed for this paper without presenting the proofs of the results presented in this paper, since they are very well deduced in the monograph Analysis on symmetric cones of Faraut and Korányi, see [2].

Let $\mathcal{A}$ be a vector space of finite dimension over a field $\mathcal{K}$ with the bilinear mapping $\bullet:(u, v) \mapsto u \bullet v$ from $\mathcal{A} \times \mathcal{A}$ to $\mathcal{A}$. Then, $\mathcal{A}$ is called a power associative algebra if for any $x \in \mathcal{A}$ the subalgebra of $\mathcal{A}$ spanned by the powers of $x$ is associative. If for all $u$ and $v$ in $\mathcal{A}$ we have $\left(J_{1}\right) u \bullet v=v \bullet u$ and $\left(J_{2}\right) u \bullet\left(u^{2} \bullet v\right)=u^{2} \bullet(u \bullet v)$, with $u^{2}=u \bullet u$, then $\mathcal{A}$ is called a Jordan algebra.

If $\mathcal{A}$ is a Jordan algebra with unit element, then $\mathcal{A}$ is power associative (cf. [2]).

Example 1. Let $n$ be a natural number and $A$ and $B$ be two real matrices of order $n$. Then it is well known that in general $A B \neq B A$ where $A B$ and $B A$ represents the usual products of the matrices $A$ by $B$ and the usual product of the matrix $B$ by $A$. Nevertheless, the multiplication - define such that

$$
A \bullet B=\frac{1}{2}(A B+B A)
$$

for all real matrices $A$ and $B$ of order $n$ verifies $A \bullet B=B \bullet A$. Now, we will show that, for any two real square matrices $\boldsymbol{A}$ and $\boldsymbol{B}$ of order $n$, we have $A \bullet\left(A^{2} \bullet B\right)=A^{2} \bullet(A \bullet B)$. Indeed, we have that

$$
\begin{aligned}
4 A \bullet\left(A^{2} \bullet B\right) & =A\left(A^{2} B+B A^{2}\right)+\left(A^{2} B+B A^{2}\right) A \\
& =A\left(A^{2} B\right)+A\left(B A^{2}\right)+\left(A^{2} B\right) A+\left(B A^{2}\right) A \\
& =A^{2}(A B)+(A B) A^{2}+A^{2}(B A)+(B A) A^{2} \\
& =A^{2}(A B+B A)+(A B+B A) A^{2} \\
& =4 A^{2} \bullet(A \bullet B) .
\end{aligned}
$$

And, therefore we have $A \bullet\left(A^{2} \bullet B\right)=A^{2} \bullet(A \bullet B)$. Hence, the vector space $\mathcal{V}=\mathcal{M}_{n}(\mathbb{R})$ over the field of the real numbers with the operation $\bullet$ is a Jordan algebra. But, we must also say that with this operation, $\bullet$, the square of a real matrix of order $n$ is such that $A \bullet A=A A$ where $A A$ represents the usual product of matrices. One proves by induction that the power of order $n$ relative- 
ly to the product - of a matrix $\boldsymbol{A}$ is equal to the usual power of order $n$ of $A$. The subalgebra $\operatorname{Sym}(n, \mathbb{R})$ of $\mathcal{V}$ formed by the real symmetric matrices of order $n$ of $\mathcal{V}$ with the operation - is also a Jordan algebra.

Remark 1. If $\mathcal{V}$ is a vector space over a field $\mathcal{K}$ with characteristic distinct from 2 with the operation $\star$ from $\mathcal{V} \times \mathcal{V}$ onto $\mathcal{V}$ such that $\mathcal{V}$ with this operation is an associative algebra, then $\mathcal{V}$ becomes a Jordan algebra when equipped with the operation $\bullet$ such that $^{1} x \bullet y=\frac{x \star y+y \star x}{2}$.

From now on, we only deal with real Jordan algebras with finite dimension and with unit and we will denote it by $\boldsymbol{e}$, and when we say let $\mathcal{A}$ be a Jordan algebra we are meaning that we are saying that $\mathcal{A}$ is a real Jordan algebra with the element $\boldsymbol{e}$ and is of finite dimension.

Let $\mathcal{A}$ be a Jordan algebra with the operation - of multiplication of two elements of $\mathcal{A}$ with unit element $e$. If there is an inner product $\langle\cdot, \cdot\rangle$ on $\mathcal{A}$ such that $\langle u \bullet v, w\rangle=\langle v, u \bullet w\rangle$, for any $u, v, w$ in $\mathcal{A}$ then $\mathcal{A}$ is called an Euclidean Jordan algebra.

Sometimes, we will say let $\mathcal{A}$ be an Euclidean Jordan algebra and we will use the notation $x \bullet y$ to represent the product of the elements $x$ and $y$ and $\langle x, y\rangle$ to represent the inner product of $x$ and $y$. Or, we will say let $(A, \bullet,\langle-,-\rangle, \boldsymbol{e})$ be an Euclidean Jordan algebra.

Remark 2. Let $\mathcal{A}$ be an algebra over a field $\mathbb{K}$ equipped the operation of multiplication - from $\mathcal{A} \times \mathcal{A}$ to $\mathcal{A}$ and with a inner product $\langle-,-\rangle$. Sometimes one defines for any $u \in \mathcal{A}$ the linear operator $L(u) v=u \bullet v, \forall v \in \mathcal{A}$ to express that $(\mathcal{A}, \bullet,<,>)$ is an Euclidean Jordan algebra if and only if $L(x) y=L(y) x, \forall x, y \in \mathcal{A} \quad$ and $\quad L(x) L\left(x^{2}\right)=L\left(x^{2}\right) L(x), \forall x \in \mathcal{A} \quad$ and $\langle L(x) y, z\rangle=\langle y, L(x) z\rangle, \forall x, y, z \in \mathcal{A}$.

Some examples of Euclidean Jordan algebras over the real numbers are:

1) the $\mathrm{n}$ dimensional Euclidean Jordan algebra $\mathcal{A}=\mathbb{R}^{n}$ endowed with the product $x \bullet y$ and $\langle x, y\rangle$ defined in the following way: for

$$
\begin{gathered}
x=\left(x_{1}, x_{2}, \cdots, x_{n}\right) \in \mathbb{R}^{n} \text { and for } y=\left(y_{1}, y_{2}, \cdots, y_{n}\right) \in \mathbb{R}^{n} \\
x \circ y=\left(x_{1}, x_{2}, \cdots, x_{n}\right) \bullet\left(y_{1}, y_{2}, \cdots, y_{n}\right)=\left(x_{1} y_{1}, x_{2} y_{2}, \cdots, x_{n} y_{n}\right)
\end{gathered}
$$

and $\langle x, y\rangle=\sum_{i=1}^{n} x_{i} y_{i}$. The unit element of $\mathcal{A}$ is $\boldsymbol{e}=(1,1, \cdots, 1)$.

2) the Euclidean Jordan algebra $\mathcal{A}=\mathbb{R}^{n}$, with $n \geq 2$ and with the operations $x \bullet y$ and $\langle x, y\rangle$ defined in the following way: for $x=\left(x_{1}, x_{2}, \cdots, x_{n}\right)$ and $y=\left(y_{1}, y_{2}, \cdots, y_{n}\right)$,

$$
x \bullet y=\left(x^{\mathrm{T}} y, x_{1} y_{2}+y_{1} x_{2}, x_{1} y_{3}+y_{1} x_{3}, \cdots, x_{1} y_{n}+y_{1} x_{n}\right)
$$

and $\langle x, y\rangle=\sum_{i=1}^{n} x_{i} y_{i}$. The unit element of $\mathcal{A}$ is $\boldsymbol{e}=(1,0, \cdots, 0)$.

3) the Euclidean Jordan algebra of real symmetric matrices of order $n$, $\mathcal{A}=\operatorname{Sym}(n, \mathbb{R})$ endowed with the Jordan product $x \bullet y=\frac{x y+y x}{2}$ and the ${ }^{1}$ We must note that $x \bullet y \in \mathcal{V}$, since we have $\frac{(x+y)^{2}-x^{2}-y^{2}}{2}=\frac{(x+y) \star(x+y)-x \star x-y \star y}{2}=\frac{x \star y+y \star x}{2}$ 
inner product defined by $\langle x, y\rangle=\operatorname{tr}(x \bullet y)$, where tr denotes the usual trace of matrices. The unit element of $\mathcal{A}$ is the identity matrix of order $n, I_{n}$.

4) The Euclidean Jordan algebra of self adjoint complex matrices of order $n$, $H_{n}(C)$ with

$$
x \bullet y=\frac{x y+y x}{2}
$$

and $\langle x, y\rangle=\operatorname{Re}(\operatorname{tr}(x \bullet y))$ for $x$ and $y \in H_{n}(C)$.

5) the Euclidean Jordan algebra of self adjoint quaternionic matrices of order $n, H_{n}(H)$ with

$$
x \bullet y=\frac{x y+y x}{2}
$$

and $\langle x, y\rangle=\operatorname{Re}(\operatorname{tr}(x \bullet y))$ for $x$ and $y \in H_{n}(H)$.

6) the Euclidean Jordan algebra of self adjoint octonionic matrices of order 3 , $\mathrm{H}_{3}(\mathrm{O})$ with

$$
x \bullet y=\frac{x y+y x}{2}
$$

and $\langle x, y\rangle=\operatorname{Re}(\operatorname{tr}(x \bullet y))$ for $x$ and $y \in H_{3}(O)$.

From now on, we will suppose that when we say let $\mathcal{A}$ be an Euclidean Jordan algebra we suppose that $\mathcal{A}$ is a real finite dimensional Euclidean Jordan algebra with unit element, that we will denote by $e$ and for a more explicit writing sometimes we will say let $(\mathcal{A}, \bullet,\langle-,-\rangle, \boldsymbol{e})$ be an Euclidean Jordan algebra for meaning that $\mathcal{A}$ is an Euclidean Jordan algebra with the operation - of two elements of $\mathcal{A}$ and equipped with the inner product $\langle-,-\rangle$ and that $\mathcal{A}$ has the unit element $e$.

Let consider the Euclidean Jordan algebra $(\mathcal{A}, \bullet,\langle-,-\rangle, \boldsymbol{e})$. An element $b \in \mathcal{A}$ is an idempotent if $b^{2}=b$. Two idempotents $c$ and $d$ in $\mathcal{A}$ are orthogonal if $c \bullet d=0$. Herein, we must say that if two elements of the Euclidean Jordan algebra $\mathcal{A}$ are orthogonal relatively to the product $\bullet$ of the Euclidean Jordan algebra $\mathcal{A}$ then they are also orthogonal relatively to the inner product of this Euclidean Jordan algebra. Indeed, if $x \bullet y=0$ then we have

$$
\langle x, y\rangle=\langle x, y \bullet \boldsymbol{e}\rangle=\langle y \bullet x, \boldsymbol{e}\rangle=\langle 0, \boldsymbol{e}\rangle=0 .
$$

Let $l$ be a natural number. The set $\left\{f_{1}, f_{2}, \cdots, f_{l}\right\}$ is a complete system of orthogonal idempotents if the following three conditions hold: 1) $f_{i}^{2}=f_{i}$, for $i=1, \cdots, l, 2) \quad f_{i} \bullet f_{j}=0$, if $i \neq j$, and 3) $\sum_{i=1}^{l} f_{i}=\boldsymbol{e}$. An idempotent $f$ is primitive if it is a non-zero idempotent of $\mathcal{A}$ and cannot be written as a sum of two orthogonal non-zero idempotents. We say that $\left\{f_{1}, f_{2}, \cdots, f_{l}\right\}$ is a Jordan frame if $\left\{f_{1}, f_{2}, \cdots, f_{l}\right\}$ is a complete system of orthogonal idempotents such that each idempotent is primitive.

Example 2. Let consider the Euclidean Jordan algebra $\mathcal{A}=\operatorname{Sym}(3, \mathbb{R})$ with the Jordan product

$$
x \bullet y=\frac{x y+y x}{2}, \forall x, y \in \mathcal{A}
$$


and the inner product $\langle x, y\rangle=\operatorname{tr}(x \bullet y)$. We will show that

$$
\mathcal{S}=\{c, d\}=\left\{\left[\begin{array}{lll}
1 & 0 & 0 \\
0 & 1 & 0 \\
0 & 0 & 0
\end{array}\right],\left[\begin{array}{lll}
0 & 0 & 0 \\
0 & 0 & 0 \\
0 & 0 & 1
\end{array}\right]\right\}
$$

is a complete system of orthogonal idempotents of $\mathcal{A}$. First, we must note that

$$
x^{2}=x \bullet x=\frac{x x+x x}{2}=x x=x^{2} .
$$

So the Jordan square of an element of $\mathcal{A}$ is the usual square of a symmetric matrix. Now since $c^{2}=c, d^{2}=d, c \bullet d=0$ and $c+d=I_{3}$, then $\mathcal{S}$ is a complete system of orthogonal idempotents of $\mathcal{A}$, but $S$ is not a Jordan frame of $\mathcal{A}$ since $c=f+g$ with

$$
f=\left[\begin{array}{lll}
1 & 0 & 0 \\
0 & 0 & 0 \\
0 & 0 & 0
\end{array}\right] \text { and } g=\left[\begin{array}{ccc}
0 & 0 & 0 \\
0 & 1 & 0 \\
0 & 0 & 0
\end{array}\right]
$$

and $f^{2}=f$ and $g^{2}=g$ and

$$
f \bullet g=\left[\begin{array}{lll}
1 & 0 & 0 \\
0 & 0 & 0 \\
0 & 0 & 0
\end{array}\right] \bullet\left[\begin{array}{lll}
0 & 0 & 0 \\
0 & 1 & 0 \\
0 & 0 & 0
\end{array}\right]=\left[\begin{array}{lll}
0 & 0 & 0 \\
0 & 0 & 0 \\
0 & 0 & 0
\end{array}\right] .
$$

Let consider the Euclidean Jordan algebra $(\mathcal{A}, \bullet,\langle-,-\rangle, \boldsymbol{e})$. The rank of an element $x$ in $\mathcal{A}$ is the least natural number $l$, such that the set $\left\{e, x, \cdots, x^{l}\right\}$ is linearly dependent (where $x^{2}=x \bullet x$ and $x^{k}=x \bullet x^{k-1}$ ), and we write $\operatorname{rank}(x)=l$. This concept is expanded by defining the rank of the algebra $\mathcal{A}$ as being the natural number

$$
r=\operatorname{rank}(\mathcal{A})=\max \{\operatorname{rank}(u): u \in \mathcal{A}\}
$$

The elements of $\mathcal{A}$ with rank equal to the rank of $\mathcal{A}$ are the regular elements of $\mathcal{A}$. This set of the regular elements of $\mathcal{A}$ is an open and dense set in $\mathcal{A}$. If $u$ is a regular element of $\mathcal{A}$, with $r=\operatorname{rank}(u)$, then the set $\left\{e, u, u^{2}, \cdots, u^{r}\right\}$ is linearly dependent and the set $\left\{e, u, u^{2}, \cdots, u^{r-1}\right\}$ is linearly independent. Thus, we may conclude that there exist unique real numbers $a_{1}(u), \cdots, a_{r}(u)$, such that

$$
u^{r}-a_{1}(u) u^{r-1}+\cdots+(-1)^{r} a_{r}(u) e=0
$$

where 0 is the null vector of $\mathcal{A}$. Making the necessary adjustments we obtain the polynomial in $\lambda, p(u,-)$ such that

$$
p(u, \lambda)=\lambda^{r}-a_{1}(u) \lambda^{r-1}+\cdots+(-1)^{r} a_{r}(u) .
$$

The polynomial $p(u,-)$ is called the characteristic polynomial of $u$, where each coefficient $a_{i}$ is a homogeneous polynomial of degree $i$ in the coordinates of $u$ in a fixed basis of $\mathcal{A}$. Although the characteristic polynomial $p(u,-)$ is defined for a regular element of $\mathcal{A}$, we can extend the definition of characteristic polynomial to all elements of $\mathcal{A}$ by continuity since each polynomial $a_{i}$ is 
homogeneous and the set of regular elements of $\mathcal{A}$ is dense in $\mathcal{A}$. Herein, we must say that the characteristic polynomial of a regular element of $\mathcal{A}$ coincides with his minimal polynomial and for a non regular element the minimal polynomial of this element divides its characteristic polynomial.

Herein, we will justify the reason to call the polynomial $p(x,-)$ the characteristic polynomial of $x$ when $x$ is regular. Let $x$ be a regular element of the Euclidean Jordan algebra $\mathcal{A}, L_{0}(x)$ represent the restriction of the operator $L(x)$ to the space $\mathbb{R}[x]$ and let $r=\operatorname{rank}(A)$. Now since $x$ is a regular element of $\mathcal{A}$ then we can say that $\mathcal{B}=\left\{e, x^{2}, x^{3}, \cdots, x^{r-1}\right\}$ is a basis of $\mathbb{R}[x]$. Now, let express the images of the elements of the basis $\mathcal{B}$ by the linear application $L_{0}(x)$ on the basis $\mathcal{B}$. Hence, we present the following calculations:

$$
\begin{aligned}
& L_{0}(x)(\boldsymbol{e})=x=0 \boldsymbol{e}+1 x+\cdots+0 x^{r-1} \\
& L_{0}(x) x=x^{2}=0 \boldsymbol{e}+0 x+1 x^{2}+\cdots+0 x^{r-1} \\
& L_{0}(x)\left(x^{2}\right)=x^{3}=0 e+0 x+0 x^{2}+1 x^{3}+\cdots+0 x^{r-1} \\
& L_{0}(x)\left(x^{r-2}\right)=x^{r-1}=0 \boldsymbol{e}+0 x+0 x^{2}+\cdots+1 x^{r-1} \\
& L_{0}\left(x^{r-1}\right)=x^{r}=-(-1)^{r} a_{r}(x) \boldsymbol{e}-(-1)^{r-1} a_{r-1}(x) x-\cdots+a_{1}(x) x^{r-1} .
\end{aligned}
$$

Therefore the matrix of the operator $L_{0}(x)$ on the basis $\mathcal{B}$ is

$$
\begin{aligned}
M_{L_{0}(x)} & =\left[\begin{array}{cccccc}
0 & 0 & 0 & \cdots & 0 & -(-1)^{r} a_{r}(x) \\
1 & 0 & 0 & \cdots & 0 & -(-1)(-1)^{r-1} a_{r-1}(x) \\
0 & 1 & 0 & \cdots & 0 & -(-1)^{r-2} a_{r-2}(x) \\
\vdots & \vdots & \vdots & \ddots & \vdots & \vdots \\
0 & 0 & 0 & \cdots & 1 & a_{1}(x)
\end{array}\right] \\
& =\left[\begin{array}{cccccc}
0 & 0 & 0 & \cdots & 0 & -(-1)^{r} a_{r}(x) \\
1 & 0 & 0 & \cdots & 0 & 0-(-1)^{r-1} a_{r-1}(x) \\
0 & 1 & 0 & \cdots & 0 & 0 \\
\vdots & \vdots & \vdots & \ddots & \vdots & \vdots \\
0 & 0 & 0 & \cdots & 1 & -\left(-a_{1}(x)\right)
\end{array}\right] .
\end{aligned}
$$

Now, we have

$$
\left|\lambda I_{r}-M_{L_{0}(x)}\right|=\left|\begin{array}{cccccc}
\lambda & 0 & 0 & \cdots & 0 & -(-1)^{r} a_{r}(x) \\
1 & \lambda & 0 & \cdots & 0 & -(-1)^{r-1} a_{r-1}(x) \\
0 & 1 & \lambda & \cdots & 0 & -(-1)^{r-2}(x) a_{r-2}(x) \\
\vdots & \vdots & \vdots & \ddots & \vdots & \vdots \\
0 & 0 & 0 & \cdots & 1 & \lambda-a_{1}(x)
\end{array}\right|=p(x, \lambda) .
$$

The roots of the characteristic polynomial of $x, p(x,-), \lambda_{1}, \lambda_{2}, \cdots, \lambda_{r-1}$ and $\lambda_{r}$, are called the eigenvalues of $x$. Furthermore, the coefficients $a_{1}(x)$ and $a_{r}(x)$ of the characteristic polynomial of $x$, are called the trace and the determinant of $x$, respectively. We most note that when $x$ is regular element of $\mathcal{A}$ 
then $\operatorname{trace}\left(M_{L_{0}(x)}\right)=a_{1}(x)$ and that $\left|M_{L_{0}(x)}\right|=a_{r}(x)$.

Remark 3. For a clear understanding of the theory of Euclidean Jordan algebras a very good readable survey is the chapter 5, An Introduction to Formally Real Jordan Algebras and Their Applications in Optimization written by Farid Alizadeh in the book [20].

Theorem 1. ([2], p. 43). Let $\mathcal{A}$ be a real Euclidean Jordan algebra. Then for $u$ in $\mathcal{A}$ there exist unique real numbers $l \in \mathbb{N}, \lambda_{1}, \lambda_{2}, \cdots, \lambda_{l}$, all distinct, and a unique complete system of orthogonal idempotents $\left\{f_{1}, f_{2}, \cdots, f_{l}\right\}$ such that

$$
u=\lambda_{1} f_{1}+\lambda_{2} f_{2}+\cdots+\lambda_{l} f_{l} .
$$

The numbers $\lambda_{j}$ 's of (2) are the eigenvalues of $u$ and the decomposition (2) is the first spectral decomposition of $u$.

Example 3. Let $A$ be a matrix of the Euclidean Jordan algebra $\mathcal{A}=\operatorname{Sym}(n, \mathbb{R})$ If $A$ is a matrix with $I$ distinct eigenvalues $\lambda_{1}, \lambda_{2}, \cdots, \lambda_{l-1}$ and $\lambda_{l}$, then the complete system of orthogonal idempotents associated to $A$ is the set $\mathcal{S}=\left\{f_{1}, f_{2}, \cdots, f_{l}\right\}$ where each idempotents $f_{i}$ for $i=1, \cdots, l$ is the projector on the eigenvector space of $A$ associate to the eigenvalue $\lambda_{i}$ defined by the equalities (3)

$$
f_{i}=\frac{\prod_{j=1, j \neq i}^{l}\left(\lambda_{j} I_{n}-A\right)}{\prod_{j=1, j \neq i}^{l}\left(\lambda_{j}-\lambda_{i}\right)}
$$

for $i=1, \cdots, l$. We must say, that they are unique. We first note that since $f_{i} \mathrm{~s}$ are projectors then we have $I_{n}=f_{1}+f_{2}+\cdots+f_{l}, f_{i} f_{j}=0, \forall i \neq j, i, j \in\{1, \cdots, l\}$ then $f_{i} \bullet f_{j}=0$ and $f_{i}^{2}=f_{i}$ for $i=1, \cdots, l$ and we have $A=\sum_{i=1}^{l} \lambda_{i} f_{i}$ is the first spectral decomposition associated to $A$.

But there is another way, for a clear exposition of this method of calculation see [20], to construct the idempotents $f_{i}$ 's on a more practically way. So will describe the method of construction of the idempotents. We have $A^{j}=\sum_{i=1}^{l} \lambda_{i}^{j} f_{i}, \forall j=0, \cdots, l-1$. So we obtain the following system

$$
\left\{\begin{array}{l}
I_{n}=f_{1}+f_{2}+\cdots+f_{l} \\
A=\lambda_{1} f_{1}+\lambda_{2} f_{2}+\cdots+\lambda_{l} f_{l} \\
A^{2}=\lambda_{1}^{2} f_{1}+\lambda_{2}^{2} f_{2}+\cdots+\lambda_{l}^{2} f_{l} \\
\vdots \\
A^{l-1}=\lambda^{l-1} f_{1}+\lambda_{2}^{l-1} f_{2}+\cdots+\lambda_{l}^{l-1} f_{l}
\end{array}\right.
$$

Since the matrix of the coefficients of the system (4), considering $f_{i}$ for $i=1, \cdots, l$ as unknowns and $I_{n}, A, A^{2}$ and $A^{l-1}$ as constants, is a non singular matrix since this is the transpose of the Vandermond matrix ${ }^{2}$, then using the Crammer rule we obtain that

$$
\begin{aligned}
& { }^{2} \text { We must note that } \\
& \left|\begin{array}{ccccccc}
1 & \cdots & 1 & 1 & 1 & \cdots & 1 \\
\lambda_{1} & \cdots & \lambda_{i-1} & \lambda_{i} & \lambda_{i+1} & \cdots & \lambda_{l} \\
\vdots & \ddots & \vdots & \vdots & \vdots & \ddots & \vdots \\
\lambda_{1}^{l-1} & \cdots & \lambda_{i-1}^{l-1} & \lambda_{i}^{l-1} & \lambda_{i+1}^{l-1} & \cdots & \lambda_{l}^{l-1}
\end{array}\right|=\prod_{i, j \in\{1, \cdots, l, j>i}\left(\lambda_{j}-\lambda_{i}\right) .
\end{aligned}
$$




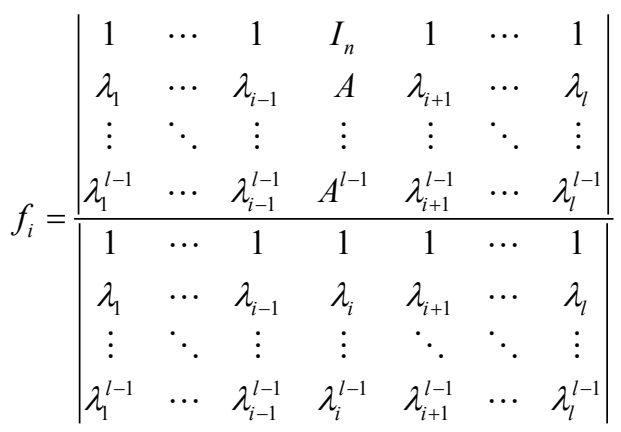

for $i=1, \cdots, l$.

Let now present a practical example. Let consider an element of the Euclidean Jordan algebra $\mathcal{A}=\operatorname{Sym}(3, \mathbb{R}), \quad A=\left[\begin{array}{lll}1 & 2 & 0 \\ 2 & 1 & 0 \\ 0 & 0 & 1\end{array}\right]$. We have that the characteristic polynomial of $A$ is $p_{A}(\lambda)=\left|\lambda I_{3}-A\right|=(\lambda-1)(\lambda+1)(\lambda-3)$. Let consider the notation $\lambda_{1}=1, \lambda_{2}=-1$ and $\lambda_{3}=3$. Now a complete system of orthogonal idempotents associated to $A$ is $\mathcal{S}=\left\{f_{1}, f_{2}, f_{3}\right\}$ with

$$
\begin{aligned}
& f_{1}=\frac{\left|\begin{array}{ccc}
I_{3} & 1 & 1 \\
A & -1 & 3 \\
A^{2} & 1 & 9
\end{array}\right|}{\left|\begin{array}{ccc}
1 & 1 & 1 \\
1 & -1 & 3 \\
1 & 1 & 9
\end{array}\right|}=\frac{-12 I_{3}-8 A+4 A^{2}}{-16}=\left[\begin{array}{lll}
0 & 0 & 0 \\
0 & 0 & 0 \\
0 & 0 & 1
\end{array}\right], \\
& f_{2}=\frac{\left|\begin{array}{ccc}
1 & I_{3} & 1 \\
1 & A & 3 \\
1 & A^{2} & 9
\end{array}\right|}{\left|\begin{array}{ccc}
1 & 1 & 1 \\
1 & -1 & 3 \\
1 & 1 & 9
\end{array}\right|}=\frac{-6 I_{3}+8 A-2 A^{2}}{-16}=\left[\begin{array}{ccc}
\frac{1}{2} & -\frac{1}{2} & 0 \\
-\frac{1}{2} & \frac{1}{2} & 0 \\
0 & 0 & 0
\end{array}\right] \text {, } \\
& f_{3}=\frac{\left|\begin{array}{ccc}
1 & 1 & I_{3} \\
1 & -1 & A \\
1 & 1 & A^{2}
\end{array}\right|}{\left|\begin{array}{ccc}
1 & 1 & 1 \\
1 & -1 & 3 \\
1 & 1 & 9
\end{array}\right|}=\frac{2 I_{3}-0 A-2 A^{2}}{-16}=\left[\begin{array}{ccc}
\frac{1}{2} & \frac{1}{2} & 0 \\
\frac{1}{2} & \frac{1}{2} & 0 \\
0 & 0 & 0
\end{array}\right] \text {. }
\end{aligned}
$$

Theorem 2. ([2], p. 44). Let $\mathcal{A}$ be a real Euclidean Jordan algebra with $\operatorname{rank}(\mathcal{A})=r$. Then for each $u$ in $\mathcal{A}$ there exists a Jordan frame $\left\{f_{1}, f_{2}, \cdots, f_{r}\right\}$ and real numbers $\lambda_{1}, \cdots, \lambda_{r-1}$ and $\lambda_{r}$ such that

$$
u=\lambda_{1} f_{1}+\lambda_{2} f_{2}+\cdots+\lambda_{r} f_{r} .
$$

The decomposition (5) is called the second spectral decomposition of $u$.

Remark 4. Now, let suppose that the $\mathrm{n}$-finite dimensional real Euclidean Jordan algebra $\mathcal{A}$ verifies $\operatorname{rank}(\mathcal{A})=n$. Since the set of regular elements of $\mathcal{A}$ 
is dense in $\mathcal{A}$, then let $x$ be one of his regular elements. So, there exists a unique complete system of orthogonal idempotents $\mathcal{S}=\left\{f_{1}, f_{2}, \cdots, f_{n}\right\}$ such that $x=\sum_{i=1}^{n} \lambda_{i} f_{i}$. But now we have that $\mathcal{S}$ is ${ }^{3}$ a linear independent set of $\mathcal{A}$, therefore $\mathcal{S}$ is a basis of $\mathcal{A}$.

Example 4. Let $\mathcal{A}$ be the Euclidean Jordan algebra such $\mathcal{A}=\operatorname{Sym}(n, \mathbb{R})$ equipped with the $\bullet$ operation such that $X \bullet Y=\frac{X Y+Y X}{2}$ and equipped with the inner product $\langle X, Y\rangle=\operatorname{tr}(X \bullet Y)$ and let $B$ be a symmetric matrix of $\mathcal{A}$ and let $\mathcal{S}=\left\{v_{1}, \cdots, v_{n}\right\}$ be an orthonormal basis of $\mathbb{R}^{n}$ of eigenvectors of $B$ with $B v_{i}=\lambda_{i} v_{i}$, for $i=1, \cdots, n$. We suppose that we are using the column notation, this is we consider the notation

$$
v_{i}=\left[\begin{array}{c}
v_{i 1} \\
\vdots \\
v_{i n}
\end{array}\right], \forall i=1, \cdots, n .
$$

Let consider $f_{i}=v_{i} v_{i}^{\mathrm{T}}$ for $i=1, \cdots, n$. Then $\left\{f_{1}, f_{2}, \cdots, f_{n}\right\}$ is a Jordan frame of $\mathcal{A}$. Indeed, let $i$ be a natural number less or equal $n$, we have

$$
f_{i}^{2}=f_{i} \bullet f_{i}=f_{i} f_{i}=v_{i} v_{i}^{\mathrm{T}} v_{i} v_{i}^{\mathrm{T}}=v_{i}\left(v_{i}^{\mathrm{T}} v_{i}\right) v_{i}^{\mathrm{T}}=v_{i} 1 v_{i}^{\mathrm{T}}=v_{i} v_{i}^{\mathrm{T}}=f_{i} .
$$

Let $i$ and $j$ be two natural numbers such that $1 \leq i, j \leq n$ and such that $i \neq j$. Then we have

$$
\begin{aligned}
f_{i} \bullet f_{j} & =\left(v_{i} v_{i}^{\mathrm{T}}\right) \bullet\left(v_{j} v_{j}^{\mathrm{T}}\right) \\
& =\frac{v_{i}\left(v_{i}^{\mathrm{T}} v_{j}\right) v_{j}^{\mathrm{T}}+v_{j}\left(v_{j}^{\mathrm{T}} v_{i}\right) v_{i}^{\mathrm{T}}}{2} \\
& =\frac{v_{i}(0) v_{j}^{\mathrm{T}}+v_{j}(0) v_{i}^{\mathrm{T}}}{2} \\
& =O_{n} .
\end{aligned}
$$

where $O_{n}$ is the real null matrix of order $n$.

So, we have proved that two any distinct idempotents $f_{i}$ s are orthogonal. Finally, since $\left\{v_{1}, v_{2}, \cdots, v_{n}\right\}$ is an orthonormal basis of $\mathbb{R}^{n}$ then we have $v_{1} v_{1}^{\mathrm{T}}+v_{2} v_{2}^{\mathrm{T}}+\cdots+v_{n} v_{n}^{\mathrm{T}}=I_{n}$, therefore we have $f_{1}+f_{2}+\cdots+f_{n}=I_{n}$. Hence, we have proved that $\mathcal{S}$ is a Jordan frame of the Euclidean Jordan algebra $\mathcal{A}$. Finally, since $B v_{i}=\lambda_{i} v_{i}$ for $i=1, \cdots, n$ then we have

$$
\begin{aligned}
B & =B I_{n}=B\left(f_{1}+f_{2}+\cdots+f_{n}\right) \\
& =B\left(v_{1} v_{1}^{\mathrm{T}}+v_{2} v_{2}^{\mathrm{T}}+\cdots+v_{n} v_{n}^{\mathrm{T}}\right) \\
& =\left(B v_{1}\right) v_{1}^{\mathrm{T}}+\left(B v_{2}\right) v_{2}^{\mathrm{T}}+\cdots+\left(B v_{n}\right) v_{n}^{\mathrm{T}} \\
& =\left(\lambda_{1} v_{1}\right) v_{1}^{\mathrm{T}}+\left(\lambda_{2} v_{2}\right) v_{2}^{\mathrm{T}}+\cdots+\left(\lambda_{n} v_{n}\right) v_{n}^{\mathrm{T}} \\
& =\lambda v_{1} v_{1}^{\mathrm{T}}+\lambda v_{2} v_{2}^{\mathrm{T}}+\cdots+\lambda_{n} v_{n} v_{n}^{\mathrm{T}} \\
& =\lambda_{1} f_{1}+\lambda_{2} f_{2}+\cdots+\lambda_{n} f_{n} .
\end{aligned}
$$

Then the second spectral decomposition of $B$ is $B=\sum_{i=1}^{n} \lambda_{i} f_{i}$.

${ }^{3}$ Note that any two distinct elements of $\mathcal{S}$ are orthogonal relatively to the inner product of the Euclidean Jordan algebra $\mathcal{A}$. 


\section{Some Notions on Strongly Regular Graphs}

A graph $G$ non complete and non null is a strongly regular graph with parameters $(n, k ; \lambda, \mu)$ if $G$ is $k$-regular if for any two adjacent vertices of $G$ have exactly $\lambda$ common neighbors and any two non-adjacent vertices have $\mu$ common neighbors.

We will say, sometimes that $G$ is a $(n, k ; \lambda, \mu)$ strongly regular graph if $G$ is a strongly regular graph with parameters $(n, k ; \lambda, \mu)$.

Let $G$ be a $(n, k ; \lambda, \mu)$ strongly regular graph. It is well known that the adjacency matrix of $G, A=\left[a_{i j}\right]$, that is a binary matrix of order $n$ such that $a_{i j}=1$, if the vertex $i$ is adjacent to $j$ and 0 otherwise, satisfies the equation

$$
A^{2}=k I_{n}+\lambda A+\mu\left(J_{n}-A-I_{n}\right),
$$

where $J_{n}$ is the all one matrix of order $n$. The eigenvalues of $G$, see for instance [21], are $k, \theta$ and $\tau$, where $\theta$ and $\tau$ are given by

$$
\begin{gathered}
\theta=\left(\lambda-\mu+\sqrt{(\lambda-\mu)^{2}+4(k-\mu)}\right) / 2 \text { and } \\
\tau=\left(\lambda-\mu-\sqrt{(\lambda-\mu)^{2}+4(k-\mu)}\right) / 2, \text { (see [21]). }
\end{gathered}
$$

We must observe that $G$ is a $(n, k ; \lambda, \mu)$ strongly regular graph if and only if the complement graph of $G, \bar{G}$ is a $(n, n-k-1 ; n-2 k+\mu-2, n-2 k+\lambda)$ strongly regular graph. We must also note that the multiplicities of the eigenvalues $\theta$ and $\tau$ of $\mathrm{G}$ are:

$$
m_{\theta}=\frac{|\tau| n+\tau-k}{\theta-\tau} \text { and } m_{\tau}=\frac{\theta n+k-\theta}{\theta-\tau} \text { respectively. }
$$

From now on, we will present some well known admissibility conditions on the parameters of a strongly regular graph.

We now present in Theorem 3 an admissibility relationship between the parameters of a strongly regular graph.

Theorem 3. Let $G$ be a $(n, k ; \lambda, \mu)$ strongly regular graph. Then $k(k-1-\lambda)=\mu(n-k-1)$.

L.L. Scott in [22] established the Krein admissibility conditions (6) and (7).

$$
\begin{aligned}
& (\theta+1)(k+\theta+2 \theta \tau) \leq(k+\theta)(\tau+1)^{2} \\
& (\tau+1)(k+\tau+2 \theta \tau) \leq(k+\tau)(\theta+1)^{2} .
\end{aligned}
$$

Finally, we couldn't help of presenting the admissibility conditions on the order of a strongly regular graph $G$ and on the multiplicity of each eigenvalue distinct from the regularity of $G$, known as the absolute bounds introduced by Delsarte, Goethals and Seidel [23], which we present on the inequalities (8) and (9).

$$
\begin{aligned}
& n \leq \frac{m_{\theta}\left(m_{\theta}+3\right)}{2}, \\
& n \leq \frac{m_{\tau}\left(m_{\tau}+3\right)}{2} .
\end{aligned}
$$


A strongly regular graph $G$ is called primitive if $G$ and $\bar{G}$ are connected. A strongly regular graph that is not primitive is called an imprimitive strongly regular graph. But, we must say, that a $(n, k ; \lambda, \mu)$ strongly regular graph $G$ is imprimitive if and only if $\mu=0$ or $\mu=k$, since we analyse only non complete strongly regular graphs then we can conclude that if $G$ is a primitive strongly regular graph then $0<\mu<k$. In this paper we consider only primitive strongly regular graphs. In the section 4 we present some admissibility conditions on the spectra and on the parameters of a strongly regular graph but obtained on asymptotic algebraic way.

\section{Binomial Hadamard Series and Inequalities over the Spectra of a Strongly Regular Raph}

Let $G$ be a $(n, k ; \lambda, \mu)$ strongly regular graph with $0<\mu<k<n-1$ and let $A$ be its adjacency matrix with the distinct eigenvalues, namely $k, \theta$ and $\tau$. Now let $\mathcal{A}$ be 3 dimensional real Euclidean subalgebra, with $\operatorname{rank}(A)=3$, of the Euclidean Jordan algebra $\mathcal{V}=\operatorname{Sym}(n, \mathbb{R})$ spanned by $I_{n}$ and the natural powers of $A$.

Now, we consider the unique complete system of orthogonal idempotents $\mathcal{S}=\left\{E_{1}, E_{2}, E_{3}\right\}$ of $\mathcal{A}$ associated to $A$, with

$$
\begin{gathered}
E_{1}=1 / n I_{n}+1 / n A+1 / n\left(J_{n}-A-I_{n}\right), \\
E_{2}=(|\tau| n+\tau-k) /(n(\theta-\tau)) I_{n}+(n+\tau-k) /(n(\theta-\tau)) A \\
+(\tau-k) /(n(\theta-\tau))\left(J_{n}-A-I_{n}\right)
\end{gathered},
$$

and

$$
\begin{aligned}
E_{3}= & (\theta n+k-\theta) /(n(\theta-\tau)) I_{n}+(-n+k-\theta) /(n(\theta-\tau)) A \\
& +(k-\theta) /(n(\theta-\tau))\left(J_{n}-A-I_{n}\right)
\end{aligned} .
$$

Let $i, j$ be natural numbers such that $1 \leq i, j \leq 3$ and $i \neq j$. So, since the idempotents $E_{i}$ and $E_{j}$ are orthogonal relatively to the Jordan product of matrices, then they are orthogonal relatively to the inner product

$$
\langle X, Y\rangle=\operatorname{tr}(X \bullet Y), \forall X, Y \in \mathcal{A}
$$

they are also orthogonal to the inner product $\langle X, Y\rangle_{1}=\operatorname{tr}(X Y)$, for all $X, Y \in \mathcal{A}$. Therefore we conclude that $S=\left\{E_{1}, E_{2}, E_{3}\right\}$ is a basis of $\mathcal{A}$.

Now, we will consider some notation for defining the Hadamard product and the Kronecker product of two matrices. We denote the space of real square matrices of order $n$ by $\mathcal{M}_{n}(\mathbb{R})$ and we define the Hadamard product and the Kronecker product of two matrices $E$ and $F$ of order $n$ of $\mathcal{M}_{n}(\mathbb{R})$ in the following way: if $E=\left[e_{i j}\right]$ and $F=\left[f_{i j}\right]$, then we define $E \circ F=\left[e_{i j} f_{i j}\right]$ and $E \otimes F=\left[e_{i j} F\right]$ for all $i, j \in\{1, \cdots, n\}$. For any natural number $l$ and for any matrix $H \in M_{n}(\mathbb{R})$ we define $H^{\circ l}$ like as follows: $H^{\circ 0}=J_{n}, H^{\circ 1}=H$ and for $l \geq 2$ we define $H^{\circ l}=H^{\circ(l-1)} \circ H \quad$ (see [24]). 
Let $x$ be a real number such that $0 \leq x<1$, herein we analyze different Hadamard series from the one used on the publication [9] but we use a algebraic method similar to the method that was used in the paper [9]. Let consider the binomial Hadamard series

$$
S_{x}=\sum_{j=0}^{+\infty}(-1)^{j}\left(\begin{array}{c}
-x \\
j
\end{array}\right)\left(\frac{A^{2}}{k+|\tau|}\right)^{\circ j} .
$$

Then $S_{x}=\sum_{i=1}^{3} q_{i x} E_{i}$ is the spectral decompositions of $S_{x}$ respectively to the Jordan frame $\mathcal{B}=\left\{E_{1}, E_{2}, E_{3}\right\}$ of $\mathcal{A}$. Now, we will show that the eigenvalues $q_{i x}$ s of $S_{x}$ are positive. We have

$$
(-1)^{j}\left(\begin{array}{c}
-x \\
j
\end{array}\right)=(-1)^{j} \frac{(-x)(-x-1)(-x-2) \cdots(-x-j+1)}{j !}
$$

and therefore

$$
(-1)^{j}\left(\begin{array}{c}
-x \\
j
\end{array}\right)=(-1)^{2 j} \frac{(x)(x+1)(x+2) \cdots(x+j-1)}{j !} \geq 0 .
$$

We denote the partial sum of order $n$ of the Hadamard series

$$
\sum_{j=0}^{+\infty}(-1)^{j}\left(\begin{array}{c}
-x \\
j
\end{array}\right)\left(\frac{A^{2}}{k+|\tau|}\right)^{\circ j}
$$

by $S_{n x}$. Hence

$$
S_{n x}=\sum_{j=0}^{n}(-1)^{j}\left(\begin{array}{c}
-x \\
j
\end{array}\right)\left(\frac{A^{2}}{k+|\tau|}\right)^{\circ j}
$$

and let consider the notation $S_{n x}=q_{n 1 x} E_{1}+q_{n 2 x} E_{2}+q_{n 3 x} E_{3}$.

Now we must note the eigenvalues of $\frac{A^{2}}{k+|\tau|}$ are positive and that for any two real matrices $E$ and $F$ of order n we have $\lambda_{\min }(E) \lambda_{\min }(F) \leq \lambda_{\min }(E \circ F)$, and we must also observe that since $\mathcal{S}$ is a Jordan frame of the Euclidean Jordan algebra $\mathcal{A}$ that is a basis of $\mathcal{A}$ and this Euclidean Jordan algebra is closed for the Hadamard product then we conclude that the eigenvalues of $S_{n x}$ are all positive.

Since $q_{1 x}=\lim _{n \rightarrow+\infty} q_{n 1 x}, q_{2 x}=\lim _{n \rightarrow+\infty} q_{n 3 x}, q_{3 x}=\lim _{n \rightarrow+\infty} q_{n 3 x}$ then we have $q_{1 x} \geq 0, q_{2 x} \geq 0$ and $q_{3 x} \geq 0$.

We have $S_{x} E_{1}=q_{1 x} E_{1}, S_{x} E_{2}=q_{2 x} E_{2}$ and $S_{x} E_{3}=q_{3 x} E_{3}$, and

$$
\begin{gathered}
q_{1 x}=\frac{1}{\left(1-\frac{k}{k+|\tau|}\right)^{x}}+\frac{1}{\left(1-\frac{\lambda}{k+|\tau|}\right)^{x}} k+\frac{1}{\left(1-\frac{\mu}{k+|\tau|}\right)^{x}}(n-k-1), \\
q_{2 x}=\frac{1}{\left(1-\frac{k}{k+|\tau|}\right)^{x}}+\frac{1}{\left(1-\frac{\lambda}{k+|\tau|}\right)^{x}} \theta+\frac{1}{\left(1-\frac{\theta}{k+\mu}\right)^{x}}(-\theta-1),
\end{gathered}
$$




$$
q_{3 x}=\frac{1}{\left(1-\frac{k}{k+|\tau|}\right)^{x}}+\frac{1}{\left(1-\frac{\lambda}{k+|\tau|}\right)^{x}} \tau+\frac{1}{\left(1-\frac{\mu}{k+|\tau|}\right)^{x}}(-\tau-1)
$$

By an asymptotical analysis of the eigenvalues $q_{2 x}$ and $q_{3 x}$ we establish the inequalities (10) and (20) of Theorem 4 and of Theorem 5 respectively.

Theorem 4. Let $G$ be a $(n, k ; \lambda, \mu)$-strongly regular graph with $0<\mu<k<n-1$ and with the distinct eigenvalues $k, \theta$ and $\tau$. If $\mu>\lambda$ then

$$
\frac{k+|\tau|-\mu}{|\tau|} \geq\left(\frac{k+|\tau|-\lambda}{k+|\tau|-\mu}\right)^{\theta}
$$

Proof. Let write the binomial series

$$
\sum_{j=0}^{+\infty}(-1)^{j}\left(\begin{array}{c}
-x \\
j
\end{array}\right)\left(\frac{A^{2}}{k+|\tau|}\right)^{\circ j}
$$

on the basis $\mathcal{B}=\left\{I_{n}, A, J_{n}-A-I_{n}\right\}$ of the Euclidean Jordan algebra $\mathcal{A}$.

Since $A^{2}=k I_{n}+\lambda A+\mu\left(J_{n}-A-I_{n}\right)$ then we have

$$
\begin{aligned}
S_{x}= & \sum_{j=0}^{+\infty}(-1)^{j}\left(\begin{array}{c}
-x \\
j
\end{array}\right)\left(\frac{A^{2}}{k+|\tau|}\right)^{\circ j} \\
= & \sum_{j=0}^{+\infty}(-1)^{j}\left(\begin{array}{c}
-x \\
j
\end{array}\right)\left(\frac{k}{k+|\tau|} I_{n}+\frac{\lambda}{k+|\tau|} A+\frac{\mu}{k+|\tau|}\left(J_{n}-A-I_{n}\right)\right)^{\circ j}, \\
= & \sum_{j=0}^{+\infty}(-1)^{j}\left(\begin{array}{c}
-x \\
j
\end{array}\right)\left(\frac{k}{k+|\tau|}\right)^{\circ j} I_{n}+\sum_{j=0}^{+\infty}(-1)^{j}\left(\begin{array}{c}
-x \\
j
\end{array}\right)\left(\frac{\lambda}{k+|\tau|}\right)^{\circ j} A \\
& +\sum_{j=0}^{+\infty}(-1)^{j}\left(\begin{array}{c}
-x \\
j
\end{array}\right)\left(\frac{\mu}{k+|\tau|}\right)^{\circ j}\left(J_{n}-A-I_{n}\right) .
\end{aligned}
$$

Therefore, we conclude that

$$
S_{x}=\frac{1}{\left(1-\frac{k}{k+|\tau|}\right)^{x}} I_{n}+\frac{1}{\left(1-\frac{\lambda}{k+|\tau|}\right)^{x}} A+\frac{1}{\left(1-\frac{\mu}{k+|\tau|}\right)^{x}}\left(J_{n}-A-I_{n}\right) .
$$

Since $S_{x} E_{2}=q_{2 x} E_{2}$ then

$$
q_{2 x}=\frac{1}{\left(1-\frac{k}{k+|\tau|}\right)^{x}}+\frac{1}{\left(1-\frac{\lambda}{k+|\tau|}\right)^{x}} \theta+\frac{1}{\left(1-\frac{\mu}{k+|\tau|}\right)^{x}}(-\theta-1) .
$$

Since $q_{2 x} \geq 0$ and $\lambda<\mu$ then rewriting (13) we obtain

$$
\frac{1}{\left(1-\frac{k}{k+|\tau|}\right)^{x}}-\frac{1}{\left(1-\frac{\mu}{k+|\tau|}\right)^{x}}-\left(\frac{1}{\left(1-\frac{\mu}{k+|\tau|}\right)^{x}}-\frac{1}{\left(1-\frac{\lambda}{k+\theta}\right)^{x}}\right) \theta \geq 0 .
$$

And therefore 


$$
\frac{1}{\left(1-\frac{k}{k+|\tau|}\right)^{x}}-\frac{1}{\left(1-\frac{\mu}{k+|\tau|}\right)^{x}} \geq\left(\frac{1}{\left(1-\frac{\mu}{k+|\tau|}\right)^{x}}-\frac{1}{\left(1-\frac{\lambda}{k+|\tau|}\right)^{x}}\right) \theta
$$

After some algebraic manipulation of (14) we obtain the inequality (15).

$$
1 \geq \frac{\left(1-\frac{k}{k+|\tau|}\right)^{x}}{\left(1-\frac{\lambda}{k+|\tau|}\right)^{x}} \cdot \frac{\left(1-\frac{\lambda}{k+|\tau|}\right)^{x}-\left(1-\frac{\mu}{k+|\tau|}\right)^{x}}{\left(1-\frac{\mu}{k+|\tau|}\right)^{x}-\left(1-\frac{k}{k+|\tau|}\right)^{x}} \theta
$$

Now, applying limits as $x$ tends to zero to both hand sides of (15) we obtain (16).

$$
1 \geq \lim _{x \rightarrow 0} \frac{\left(1-\frac{k}{k+|\tau|}\right)^{x}}{\left(1-\frac{\lambda}{k+|\tau|}\right)^{x}} \frac{\left(1-\frac{\lambda}{k+|\tau|}\right)^{x}-\left(1-\frac{\mu}{k+|\tau|}\right)^{x}}{\left(1-\frac{\mu}{k+|\tau|}\right)^{x}-\left(1-\frac{k}{k+|\tau|}\right)^{x}} .
$$

Recurring to the rule of L'Hopital to the second factor of the right hand side of (16) we obtain (17)

$$
1 \geq \frac{\ln \left(1-\frac{\lambda}{k+|\tau|}\right)-\ln \left(1-\frac{\mu}{k+|\tau|}\right)}{\ln \left(1-\frac{\mu}{k+|\tau|}\right)-\ln \left(1-\frac{k}{k+|\tau|}\right)} \theta .
$$

Recurring to the properties of logarithms on the right hand side of the inequality (17) we deduce (18).

$$
1 \geq \frac{\ln \left(\frac{k+|\tau|-\lambda}{k+|\tau|-\mu}\right)}{\ln \left(\frac{k+|\tau|-\mu}{|\tau|}\right)} \theta
$$

Rewriting (18) we obtain the inequality (19)

$$
\frac{k+|\tau|-\mu}{|\tau|} \geq\left(\frac{k+|\tau|-\lambda}{k+|\tau|-\mu}\right)^{\theta} .
$$

Theorem 5. Let $G$ be a $(n, k ; \lambda, \mu)$-strongly regular graph with $0<\mu<k<n-1$ and with the distinct eigenvalues $k, \theta$ and $\tau$. If $\lambda>\mu$ then

$$
\frac{k+|\tau|-\mu}{|\tau|} \geq\left(\frac{k+|\tau|-\mu}{k+|\tau|-\lambda}\right)^{|\tau|} .
$$

Proof. Since $q_{3 x} \geq 0, \quad \mu<\lambda$ and

$$
q_{3 x}=\frac{1}{\left(1-\frac{k}{k+|\tau|}\right)^{x}}+\frac{1}{\left(1-\frac{\lambda}{k+|\tau|}\right)^{x}} \tau+\frac{1}{\left(1-\frac{\mu}{k+|\tau|}\right)^{x}}(-\tau-1) \geq 0
$$


then, we have (21).

$$
\frac{1}{\left(1-\frac{k}{k+|\tau|}\right)^{x}}-\frac{1}{\left(1-\frac{\mu}{k+|\tau|}\right)^{x}}+\left(\frac{1}{\left(1-\frac{\lambda}{k+|\tau|}\right)^{x}}-\frac{1}{\left(1-\frac{\mu}{k+|\tau|}\right)^{x}}\right) \tau \geq 0 .
$$

After some algebraic manipulation of (21) we obtain (22).

$$
\frac{1}{\left(1-\frac{k}{k+|\tau|}\right)^{x}}-\frac{1}{\left(1-\frac{\mu}{k+|\tau|}\right)^{x}} \geq\left(\frac{1}{\left(1-\frac{\lambda}{k+|\tau|}\right)^{x}}-\frac{1}{\left(1-\frac{\mu}{k+|\tau|}\right)^{x}}|| \tau \mid .\right.
$$

After a rewriting of the inequality (22) we deduce (23).

$$
1 \geq \frac{\left(1-\frac{k}{k+|\tau|}\right)^{x}}{\left(1-\frac{\lambda}{k+|\tau|}\right)^{x}} \cdot \frac{\left(1-\frac{\mu}{k+|\tau|}\right)^{x}-\left(1-\frac{\lambda}{k+|\tau|}\right)^{x}}{\left(1-\frac{\mu}{k+|\tau|}\right)^{x}-\left(1-\frac{k}{k+|\tau|}\right)^{x}}|\tau| .
$$

Applying limits as $x$ tends to zero to both hand sides of (23) we obtain inequality (24).

$$
1 \geq \lim _{x \rightarrow 0} \frac{\left(1-\frac{k}{k+\theta}\right)^{x}}{\left(1-\frac{\lambda}{k+|\tau|}\right)^{x}} \frac{\left(1-\frac{\mu}{k+|\tau|}\right)^{x}-\left(1-\frac{\lambda}{k+|\tau|}\right)^{x}}{\left(1-\frac{\mu}{k+|\tau|}\right)^{x}-\left(1-\frac{k}{k+|\tau|}\right)^{x} \mid} \mid
$$

Applying the rule of l'Hopital to the second factor of the right hand side of (24) as $x$ tends 0 we deduce (25).

$$
1 \geq \frac{\ln \left(1-\frac{\mu}{k+|\tau|}\right)-\ln \left(1-\frac{\lambda}{k+|\tau|}\right)}{\ln \left(1-\frac{\mu}{k+|\tau|}\right)-\ln \left(1-\frac{k}{k+|\tau|}\right)}|\tau| .
$$

Recurring to the properties of logarithms we deduce from (25) the inequality (26).

$$
1 \geq \frac{\ln \left(\frac{k+|\tau|-\mu}{k+|\tau|-\lambda}\right)}{\ln \left(\frac{k+|\tau|-\mu}{|\tau|}\right)}|\tau|
$$

And, finally from (26) we conclude that $\frac{k+|\tau|-\mu}{|\tau|} \geq\left(\frac{k+|\tau|-\mu}{k+|\tau|-\lambda}\right)^{|\tau|}$.

Now, considering the elements of $\mathcal{A}$, 


$$
\begin{gathered}
S_{\theta}=E_{3} \circ\left(\sum_{j=0}^{+\infty}(-1)^{j}\left(\begin{array}{c}
-x \\
j
\end{array}\right)\left(\frac{A^{2}}{k+|\tau|}\right)^{\circ j}\right) \\
\text { and } S_{\tau}=E_{3} \circ\left(\sum_{j=0}^{+\infty}(-1)^{j}\left(\begin{array}{c}
-x \\
j
\end{array}\right)\left(\frac{A^{2}}{k+\theta}\right)^{\circ j}\right)
\end{gathered}
$$

and analysing the eigenvalues $q_{1 \theta}$ and $q_{1 \tau}$ of the spectral decompositions $S_{\theta}=q_{1 \theta} E_{1}+q_{2 \theta} E_{2}+q_{3} E_{3}$ and $S_{\tau}=q_{1 \tau} E_{1}+q_{2 \tau} E_{2}+q_{3 \tau} E_{3}$ respectively, and making similars algebraic asymptotic analysis to that done on the proofs of Theorems 4 and 5 we deduce the inequalities (27) and (28) of Theorem 6.

Theorem 6. Let $G$ be a $(n, k ; \lambda, \mu)$-strongly regular graph with

$0<\mu<k<n-1$ and with the distinct eigenvalues $k, \theta$ and $\tau$. If $k<\frac{n}{2}$ and $\lambda>\mu$ then

$$
\begin{aligned}
& \left(\frac{k+|\tau|-\mu}{|\tau|}\right)^{2 \theta+1} \geq\left(\frac{k+|\tau|-\mu}{k+|\tau|-\lambda}\right)^{k}, \\
& \left(\frac{k+\theta-\mu}{\theta}\right)^{2 \theta+1} \geq\left(\frac{k+\theta-\mu}{k+\theta-\lambda}\right)^{k}
\end{aligned}
$$

\section{Acknowledgements}

Lus Vieira in this work was partially supported by the Center of Research of Mathematics of University of Porto (UID/MAT/00144/2013), which is funded by FCT (Portugal) with national (MEC) and European structural funds through the programs FEDER, under the partnership agreement PT2020.

\section{Conflicts of Interest}

The author declares no conflicts of interest regarding the publication of this paper.

\section{References}

[1] McCrimmon, K. (2000) A Taste on Jordan Algebras. Springer, New York.

[2] Faraut, J. and Korányi, A. (1994) Analysis on Symmetric Cones, Oxford Mathematical Monographs. Clarendon Press, Oxford.

[3] Koecher, M. (1999) The Minnesota Notes on Jordan Algebras and Their Applications. Springer, Berlin. https://doi.org/10.1007/BFb0096285

[4] Cardoso, D.M. and Vieira, L.A. (2006) On the Optimal Parameter of a Self-Concordant Barrier over a Symmetric Cone. European Journal of Operational Research, 169, 1148-1157. https://doi.org/10.1016/j.ejor.2004.11.027

[5] Schmieta, S.H. and Alizadeh, F. (2001) Associative and Jordan Algebras, and Polynomial time Interior-Point Algorithms for Symmetric Cones. Mathematics of Operations Research, 26, 543-564. https://doi.org/10.1287/moor.26.3.543.10582

[6] Faybusovich, L. (1997) Linear Systems in Jordan Algebras and Primal-Dual Interior-Point Algorithms. Journal of Computational and Applied Mathematics, 86, 149-175. https://doi.org/10.1016/S0377-0427(97)00153-2 
[7] Faybusovich, L. (1997) Euclidean Jordan Algebras and Interior-Point Algorithms. Positivity, 1, 331-357. https://doi.org/10.1023/A:1009701824047

[8] Jordan, P., Neuman, J.P. and Wigner, E. (1934) On an Algebraic Generalization of the Quantum Mechanical Formalism. Annals of Mathematics, 35, 29-64.

https://doi.org/10.2307/1968117

[9] Vieira, L.A. (2018) Asymptotic Properties of the Spectra of a Strongly Regular Graph, Innovation, Engineering and Entrepreneurship. Lecture notes in Electrical Engineering, Book Series, 505, 800-804.

[10] Cardoso, D.M. and Vieira, L.A. (2004) Euclidean Jordan Algebras with Strongly Regular Graphs. Journal of Mathematical Sciences, 120, 881-894. https://doi.org/10.1023/B:JOTH.0000013553.99598.cb

[11] Mano, V.M., Martins, E.A. and Vieira, L.A. (2011) On Generalized Binomial Series and Strongly Regular Graphs. Proyecciones Journal of Mathematics, 4, 393-408.

[12] Mano, V.M. and Vieira, L.A. (2011) Admissibility Conditions and Asymptotic Behaviour of Strongly Regular Graph. International Journal of Mathematical Models and Methods in Applied Sciences, 5, 1027-1033.

[13] Mano, V.M. and Vieira, L.A. (2014) Alternating Schur Series and Necessary Conditions for the Existence of Strongly Graphs. International Journal of Mathematical Models and Methods in Applied Sciences Methods, 8, 256-261.

[14] Vieira, L.A. (2009) Euclidean Jordan Algebras and Inequalities on the Parameters of a Strongly Regular Graph. AIP Conference Proceedings, 1168, 995-998.

[15] Vieira, L.A. and Mano, V.M. (2015) Generalized Krein Parameters of a Strongly Regular Graph. Applied Mathematics, 6, 37-45.

https://doi.org/10.4236/am.2015.61005

[16] Massam, H. and Neher, E. (1998) Estimation and Testing for Lattice Condicional Independence Models on Euclidean Jordan Algebras. Annals of Statistics, 26, 1051-1082. https://doi.org/10.1214/aos/1024691088

[17] Gowda, M.S. and Tao, J. (2009) Some Inertia Theorems in Euclidean Jordan Algebras. Linear Algebra and Its Applications, 430, 19991-2011.

https://doi.org/10.1016/j.laa.2008.11.015

[18] Snadjder, R., Gowda, M.S. and Moldovan, M.M. (2012) More Results on Schur Complements in Euclidean Jordan Algbras. Journal of Global Optimization, 53, 121-134. https://doi.org/10.1007/s10898-011-9734-x

[19] Gowda, M.S. (2011) Some Inequalities Involving Determinants, Eigenvalues, and Schur Complements in Euclidean Jordan Algebras. Positivity, 15, 381-399. https://doi.org/10.1007/s11117-010-0086-4

[20] Alizadeh, F. (2012) An Introduction to Formally Real Jordan Algebras and Their Applications in Optimization. In: Anjos, M.F. and Lasserre, J.B., Eds., Handbook on Semidefinite, Conic and Polynomial Optimization, Springer, New York, 297-338.

[21] Godsil, C. and Royle, G. (1993) Algebraic Graph Theory. Chapman \& Hall, New York.

[22] Scott, L.L. (1973) A Condition on Higman's Parameters. Notices of the American Mathematical Society, 20, A-97.

[23] Delsart, P., Goethals, J.M. and Seidel, J.J. (1975) Bounds for Systems of Lines and Jacobi Polynomials. Philips Research Reports, 30, 91-95.

[24] Horn, A.R. and Johnson, C.R. (1991) Topics in Matrix Analysis. Cambridge University Press, Cambridge. 